\title{
Avaliação epidemiológica da Doença de Chagas em uma comunidade rural de Paulo
}

\section{Afonso - Bahia}

\author{
Epidemiological evaluation of Chagas Disease in a rural Community of Paulo Afonso - Bahia \\ Evaluación epidemiológica de la Enfermedad de Chagas en una comunidad rural de Paulo Afonso - \\ Bahia
}

Recebido: 26/08/2021 | Revisado: 02/09/2021 | Aceito: 04/09/2021 | Publicado: 06/09/2021

Yngrid da Rocha Fernandes

ORCID: https://orcid.org/0000-0003-1502-848X

Universidade Federal do Vale do São Francisco, Brasil

E-mail: yngridrfernandes@gmail.com

Caroline Cruvinel de Souza

ORCID: https://orcid.org/0000-0002-4636-5641

Universidade Federal do Vale do São Francisco, Brasil

E-mail: cruvinelcaroline@gmail.com

Joilda Silva Nery

ORCID: https://orcid.org/0000-0002-1576-6418 Universidade Federal da Bahia, Brasil

E-mail: joildanery@gmail.com

André de Lima Aires

ORCID: https://orcid.org/0000-0001-9283-1466

Universidade Federal do Pernambuco, Brasil

E-mail: andre.laires@ufpe.br

Diogo Vilar da Fonsêca

ORCID: https://orcid.org/0000-0001-7869-7061 Universidade Federal do Vale do São Francisco, Brasil

E-mail: diogo.vilar@univasf.edu.br

Anekécia Lauro da Silva

ORCID: https://orcid.org/0000-0003-2778-1460 Universidade Federal do Vale do São Francisco, Brasil

E-mail: anekecia@gmail.com

\begin{abstract}
Resumo
O nordeste brasileiro possui elevada importância epidemiológica para Doença de Chagas (DC), uma vez que possui elevada concentração do vetor transmissor. O estado da Bahia apresenta números expressivos da endemia chagásica. Dessa maneira, esse estudo teve como objetivo traçar o perfil epidemiológico da DC em uma população rural da Bahia. Estudo de caráter quantitativo e descritivo na comunidade rural Rio do Sal, município de Paulo Afonso/Bahia. Foi utilizado aplicação de questionário epidemiológico com 64 pessoas e análise de 108 prontuários, e posteriormente, análise dos dados. A prevalência de DC encontrada foi de $0 \%$. A idade média entre homens e mulheres foi 44 anos, predominando as mulheres na pesquisa $(82,81 \%)$. As ocupações foram: aposentados $(20,32 \%)$, desempregados $(53,12 \%)$, empregados $(23,43 \%)$ e estudantes $(3,13 \%)$. Do nível de escolaridade, $(9,38 \%)$ são analfabetos; $(40,63 \%)$ possuem ensino fundamental incompleto e (12,50\%), ensino fundamental completo. Todos os indivíduos residiam em casa de alvenaria. O conhecimento sobre o vetor transmissor e a doença era insatisfatório. Quanto aos dados dos pacientes analisados em prontuário, $(35,19 \%)$ tem hipertensão arterial sistêmica (HAS) e $(7,41 \%)$, insuficiência cardíaca. No entanto, somente 9 pacientes haviam realizado o eletrocardiograma, sendo 1 alterado. Além disso, nenhum dos pacientes possuía sorologias para DC. Nesse contexto, observou-se que o perfil epidemiológico na área estudada é de um indivíduo adulto, com idade entre 40-50 anos, do sexo feminino, com baixa escolaridade, desempregado, com a HAS como principal comorbidade e sem diagnóstico para doença de Chagas.
\end{abstract}

Palavras-chave: Doença de Chagas; Trypanosoma cruzi; Epidemiologia; Ârea rural; Diagnóstico.

\begin{abstract}
The Northeast Brazil has high epidemiological importance for Chagas disease (CD), since it has high concentration of the transmitting vector. The state of Bahia presents expressive numbers of the Chagasic endemic. Thus, this study aimed to trace the epidemiological profile of $\mathrm{CD}$ in a rural population of Bahia. Quantitative and descriptive study in the rural community of Rio do Sal, Paulo Afonso / Bahia. It was used an epidemiological questionnaire with 64 people and analysis of 108 charts followed by data analysis. The prevalence of $\mathrm{CD}$ found was $0 \%$. The average age was 44 years and women predominated in the survey $(82.81 \%)$. Occupations: retirees $(20.32 \%)$, unemployed
\end{abstract}


(53.12\%), employees (23.43\%) and students (3.13\%). About the educational level, (9.38\%) are illiterate; (40.63\%) have incomplete elementary education and (12.50\%), complete elementary education. All individuals resided in the masonry house. Previous knowledge about the transmitting vector and disease was unsatisfactory. Regarding the data of patients analyzed in medical records, (35.19\%) have systemic arterial hypertension (SAH) and (7.41\%), heart failure. However, only 9 patients had performed the electrocardiogram, with 1 being altered. In addition, none of the patients had CD serology. In this context, it was observed that the epidemiological profile in the studied area is an adult individual, aged 40-50 years, female, with low educational, unemployed, with SAH as the main comorbidity and without diagnosis for Chagas' disease.

Keywords: Chagas disease; Trypanosoma cruzi; Epidemiology; Space rural; Diagnosis.

\section{Resumen}

El Nordeste Brasileño tiene una alta importancia epidemiológica para la Enfermedad de Chagas (EC), ya que tiene una alta concentración del vector transmisor. El estado de Bahía tiene un número significativo de enfermedad de Chagas endémica. Este estudio tuvo como objetivo perfilar el perfil epidemiológico de la EC en una población rural. Estudio cuantitativo y descriptivo en la comunidad rural de Rio do Sal, municipio de Paulo Afonso / Bahía. Se aplicó un cuestionario epidemiológico a 64 personas y se analizaron 108 historias clínicas, seguido del análisis de datos. La prevalencia de EC encontrada fue del $0 \%$. La edad promedio entre hombres y mujeres fue de 44 años, predominando las mujeres en la encuesta $(82,81 \%)$. Las ocupaciones fueron: jubilados $(20,32 \%)$, desempleados $(53,12 \%)$, ocupados $(23,43 \%)$ y estudiantes $(3,13 \%)$. Sobre el nível de educación, $(9,38 \%)$ son analfabetos; $(40,63 \%)$ tiene educación primaria incompleta y $(12,50 \%)$ educación primaria completa. Todos los individuos residían en una casa de ladrillos. El conocimiento sobre el vector y la enfermedad fue insatisfactorio. En relación a los datos de los pacientes analizados en la historia clínica, $(35,19 \%)$ presentaban hipertensión arterial sistémica (HAS) y $(7,41 \%)$ insuficiencia cardíaca. Sin embargo, solo a 9 pacientes se les había realizado el electrocardiograma, uno de los cuales estaba alterado. Además, ninguno de los pacientes tenía serología para EC. En este contexto, se observó que el perfil epidemiológico en el área de estudio es el de un adulto, con edad entre 40-50 años, mujer, con bajo nivel educativo, desempleado, con HAS como principal comorbilidad y sin diagnóstico de EC.

Palabras clave: Enfermedad de Chagas; Trypanosoma cruzi; Epidemiología; Meio rural; Diagnóstico.

\section{Introdução}

De grande distribuição na América Latina, a Doença de Chagas é considerada uma doença tropical negligenciada e um dos principais problemas de saúde pública do Brasil. Causada por infecção do protozoário Trypanosoma cruzi, a doença de Chagas tem evolução crônica e caracteriza-se por ser um problema resultante da pobreza, alcançando, segundo dados da OMS (Organização Mundial de Saúde), aproximadamente 16-18 milhões de pessoas infectadas no mundo, com elevados índices de morbidade e mortalidade (Dias et al., 2016; World Health Organization, 2015). Constitui-se assim, em um dos maiores desafios na saúde pública, principalmente no âmbito da promoção de saúde, que ainda necessita de melhores políticas de controle da endemia (World Health Organization, 2015; Dias, 2000a).

A doença de chagas possui duas formas clínicas definidas (aguda e crônica) (Lima, Teixeira \& Lima, 2019; Porfírio, Lobato, Trindade \& Araújo Filho, 2020). As manifestações agudas são evidenciadas em consequência da entrada do protozoário na corrente sanguínea, acarretando sintomas gerais e alterações sistêmicas (Coura, Viñas, Soares, Sousa \& Xavier, 2013; Menezes, Costa, Gollob \& Dutra, 2011). A forma crônica pode ser assintomática ou sintomática e ainda indeterminada, sendo a forma sintomática caracterizada por alterações principalmente nos sistemas digestivo e cardíaco, gerando assim níveis elevados de morbidade (World Health Organization, 2015; Coura et al., 2013).

A região Nordeste, sempre teve importância acentuada no cenário epidemiológico da doença de Chagas, isso ocorre devido a grande concentração de espécies de vetores triatomíneos nessa região (Dias, Machado, Fernandes \& Vinhaes, 2000). Atualmente estima-se que 1,9 milhões de indivíduos estejam infectados no Brasil (World Health Organization, 2015). O estado da Bahia registrou um número representativo de óbitos por DC (Amorim \& Costa, 2021). Embora a maioria dos indivíduos chagásicos apresente a forma crônica indeterminada, ainda é uma doença negligenciada que causa elevada mortalidade (Coura et al., 2013; Brasil, 2018).

Portanto, nota-se que na região do Norte da Bahia, onde está localiza a cidade de Paulo Afonso, apesar de ser região endêmica para DC com confirmação da presença de insetos triatomíneos, existem poucas informações e trabalhos publicados 
acerca da epidemiologia da doença de Chagas nessa região. O objetivo deste trabalho é estabelecer o perfil epidemiológico da DC na população moradora da comunidade Rio do Sal que são atendidos pelo Programa de Saúde da Família na unidade Rio do Sal, levando em conta os seguintes aspectos: idade, sexo, escolaridade, prevalência da infecção, ocupação, conhecimentos sobre o vetor e a doença, assim como levantamento e análise de prontuários médicos em busca de diagnósticos da doença de chagas.

\section{Metodologia}

Trata-se de um estudo de natureza retrospectiva, de caráter quantitativo e descritivo (Fontelles, Simões, Farias \& Fontelles, 2009). Realizado na comunidade rural Rio do Sal, município de Paulo Afonso- BA. Teve como local de apoio, a Unidade Básica de Saúde do Rio do Sal. Nesta unidade existe uma equipe de Saúde da Família que atende uma população de 120 mil habitantes de acordo com o Censo do instituto brasileiro de geografia estatística (IBGE). O estudo foi realizado em duas etapas. A primeira etapa consistiu na aplicação de questionário epidemiológico nas residências dos participantes da pesquisa (Bozelli, Araújo, Guilherme \& Gomes, 2006). As variáveis obtidas foram: nome, idade, sexo, escolaridade, atividade profissional, hemotransfusão, conhecimentos sobre o vetor e conhecimentos sobre a doença de Chagas, com a finalidade de apresentar os aspectos epidemiológicos da região e discutir a incidência da doença. A aplicação dos questionários foi realizada no período de maio a julho de 2018 em pessoas na faixa etária entre 18 a 85 anos. A participação na pesquisa foi voluntária, sendo antecedida pela assinatura do termo de consentimento informado e autorizada pelo Comitê de Ética da UNIVASF cujo número do processo é 84064218.0.0000.5196. Na segunda etapa do estudo foram analisados 108 prontuários de pacientes atendidos pela unidade de Saúde Rio do Sal à procura de pacientes diagnosticados com a Doença de Chagas no período de 2014 - 2016. Após isso, os dados foram analisados de forma quantitativa e descritiva (Teixeira \& Oliveira, 2015).

\section{Resultados}

\section{Dados Epidemiológicos}

Responderam ao questionário epidemiológico 64 indivíduos com idade entre 18 e 85 anos, sendo a média de idade de 44 anos. Em relação ao sexo, 82,81\% (53) eram feminino e 17,19\% (11) masculino, tendo assim o sexo feminino com maior destaque na participação da entrevista (Tabela 1). Em relação à escolaridade dos indivíduos nessa região, a maioria possuía o ensino fundamental incompleto (40,63\%/ (26); $12,50 \%$ (8) afirmaram ter ensino fundamental completo, e a quantidade de indivíduos analfabetos foi de 3,98\% (6) não sendo um número expressivo nessa população (Tabela 1). 
Tabela 1. Perfil epidemiológico de 64 indivíduos residentes na comunidade rural do Rio do Sal, pertencente ao município de Paulo Afonso (BA). Agosto de 2018.

\begin{tabular}{|c|c|}
\hline Variável & $\%(n)$ \\
\hline \multicolumn{2}{|l|}{ 1. Sexo } \\
\hline Feminino & $82,81 \%(53)$ \\
\hline Masculino & $17,19 \%(11)$ \\
\hline \multicolumn{2}{|l|}{ 2. Idade } \\
\hline $18-30$ & $9,38 \%(17)$ \\
\hline $31-50$ & $39,05 \%(25)$ \\
\hline $51-70$ & $18,75 \%(12)$ \\
\hline $71-85$ & $12,5 \%(8)$ \\
\hline \multicolumn{2}{|l|}{ 3. Escolaridade } \\
\hline Analfabeto & $9,38 \%(6)$ \\
\hline EFI & $40,63 \%(26)$ \\
\hline EFC & $12,50 \%(8)$ \\
\hline EMI & $23,44 \%(15)$ \\
\hline EMC & $14,09 \%(9)$ \\
\hline \multicolumn{2}{|l|}{ 4.Ocupação } \\
\hline Aposentados & $20,32 \%(13)$ \\
\hline Desempregados & $53,12 \%(34)$ \\
\hline Empregados & $23,43 \%(15)$ \\
\hline Estudantes & $3,13(2)$ \\
\hline \multicolumn{2}{|l|}{ 5. Zona Residencial } \\
\hline Rural & $100 \%(64)$ \\
\hline Urbana & $0 \%(0)$ \\
\hline \multicolumn{2}{|l|}{ 6. Tipo de moradia } \\
\hline Alvenaria & $100 \%(64)$ \\
\hline Taipa & $0 \%(0)$ \\
\hline \multicolumn{2}{|l|}{ 7. Sempre residiu na comunidade } \\
\hline Sim & $68,75 \%(44)$ \\
\hline Não & $31,25 \%(20)$ \\
\hline
\end{tabular}

Fonte: Dados da Pesquisa. EFI: Ensino Fundamental Incompleto, EFC: Ensino Fundamental. Completo, EMI: Ensino Médio Incompleto, EMC: Ensino Médio Completo.

Referente à ocupação dos entrevistados, 20,32\% (13) eram aposentados; 53,12\% (34) estavam desempregados; $23,43 \%$ (15) estavam empregados e 3,13\% (2) somente estudavam. Observou-se que o número de casas de alvenaria existente em Rio do Sal é significativamente expressivo 100\% (64). Foi possível verificar nessa região a modificação do tipo de casas de taipa para alvenaria, antes muito encontradas nas áreas rurais.

Quanto à zona de residência, houve prevalência da zona rural em 100\% (64) dos entrevistados. Observou-se que a maioria dos participantes da entrevista são moradores fixos da comunidade Rio do Sal desde o nascimento 68,75\% (44) e que os demais participantes $31,25 \%$ (20) residiram em outros municípios anteriormente.

Ainda na entrevista, foi avaliado o conhecimento dos indivíduos participantes sobre o vetor transmissor da Doença de Chagas (Tabela 2). Nesta avaliação 85,94\% (55) das pessoas afirmaram conhecer o barbeiro e 14,06\% (9) não conheciam; $7,81 \%$ (5) dos entrevistados relataram já terem sido picados pelo barbeiro enquanto 85,94\% (59) afirmaram nunca ter sido picado (Tabela 2). Apesar de poucas pessoas afirmarem ter sido picadas pelo barbeiro, 37,50\% (24) das pessoas relataram que já encontraram o vetor em suas residências, porém, a maioria 62,50\% (40) nunca o encontrou (Tabela 2). Embora grande parte dos moradores da área do Rio do Sal apresente conhecimento de que o barbeiro pode transmitir alguma doença 87,50\% (56), a maior parte deles não sabem como evitar o barbeiro em sua casa 95,31\% (61) (Tabela 2). 
Tabela 2. Avaliação do conhecimento da população rural da comunidade Rio do Sal (Paulo Afonso, BA) sobre o vetor transmissor da Doença de Chagas. Agosto de 2018.

\begin{tabular}{lc}
\hline \multicolumn{1}{c}{ Variáveis } & $\%(\mathbf{n})$ \\
\hline 1.Conhece o Barbeiro? & $85,94 \%(55)$ \\
Sim & $14,06 \%(9)$ \\
Não & $7,81 \%(5)$ \\
& \\
2.Já foi picado pelo barbeiro? & $92,19 \%(59)$ \\
Sim & \\
Não & $37,50 \%(24)$ \\
3.Já encontrou o barbeiro em sua residência ou próximo de sua casa? & $62,50 \%(40)$ \\
Sim & \\
Não & $4,69 \%(3)$ \\
4.Sabe como evitar o em sua casa? & $95,31 \%(61)$ \\
Sim & \\
Não & $87,50 \%(56)$ \\
5.Acha que o barbeiro transmite doença? & $12,50 \%(8)$ \\
Sim & \\
Não & \\
\hline
\end{tabular}

Fonte: Dados da pesquisa.

Quanto ao nível de conhecimento da população do Rio do Sal acerca da Doença de Chagas, 46,88\% (30) dos participantes sabiam responder o que é a doença de Chagas, enquanto 53,13\% (34) não tinham conhecimento sobre a doença (Tabela 3). Em relação ao modo de transmissão dessa parasitose, 39,06\% (25) dos indivíduos afirmaram saber como a DC é transmitida ao homem e 60,94\% (39) dos participantes não sabiam como ocorria a transmissão (Tabela 3). Embora na entrevista tenham existido afirmações positivas acerca da ocorrência de picada do barbeiro e também da presença do mesmo em local residencial (Tabela 2), não há histórico de DC nas famílias dos participantes 64(100\%) (Tabela 3). Com referência a transfusão de sangue, uma das formas de transmissão da DC, somente 4,69\% (3) das pessoas afirmaram já ter recebido.

Tabela 3. Avaliação do conhecimento da população rural da comunidade Rio do Sal (Paulo Afonso, BA) sobre a Doença de Chagas. Agosto de 2018.

\begin{tabular}{lc}
\hline \multicolumn{1}{c|}{ Variáveis } & $\%(\mathbf{n})$ \\
\hline 1. Sabe o que é a Doença de Chagas? & $46,88 \%(30)$ \\
Sim & $53,13 \%(34)$ \\
Não & \\
2. Sabe como pega a Doença de Chagas? & $39,06 \%(25)$ \\
Sim & $60,94 \%(39)$ \\
Não & $0 \%(0)$ \\
3.Alguém em sua casa tem a Doença de Chagas? & $100 \%(64)$ \\
Sim & \\
Não & $4,69 \%(3)$ \\
4. Já recebeu transfusão de sangue? & $95,31 \%(61)$ \\
Sim & \\
Não &
\end{tabular}

Fonte: Dados da pesquisa. 


\section{Análise de Prontuários}

Foram analisados 108 prontuários, com faixa etária de maior prevalência de 31-50 anos 45,36\% (49). Dos prontuários analisados, $58,33 \%$ (63) eram do sexo feminino e $41,67 \%$ (45) do sexo masculino. Como pode ser observado na tabela 4 , o sexo feminino 58,33\% (63) predominou entre os pacientes atendidos pela unidade de saúde do Rio do Sal. Em nenhum dos prontuários analisados constava solicitação ou resultado de sorologia para a doença de Chagas. Em 35,19\% (38) dos pacientes foi identificada a presença de comorbidades como a Hipertensão Arterial Sistêmica (HAS) e a Insuficiência Cardíaca (IC), sendo que dos 38 Hipertensos 7,41\% (8) possuíam IC (Tabela 4). As principais doenças associadas foram a diabetes mellitus, e a doença arterial coronária.

Tabela 4: Análise de 108 prontuários de indivíduos atendidos na Unidade Básica de Saúde Rio do Sal, localizada na comunidade rural Rio do Sal (Paulo Afonso/ Bahia). Agosto de 2018.

\begin{tabular}{|c|c|}
\hline Variável & $\%(n)$ \\
\hline \multicolumn{2}{|l|}{ 1. Sexo } \\
\hline Feminino & $58,33 \%(63)$ \\
\hline Masculino & $41,67 \%(45)$ \\
\hline \multicolumn{2}{|l|}{ 2. Idade } \\
\hline $18-30$ & $11,08 \%(12)$ \\
\hline $31-50$ & $45,36 \%(49)$ \\
\hline $51-70$ & $27,8 \%(30)$ \\
\hline $71-85$ & $15,76 \%(17)$ \\
\hline \multicolumn{2}{|l|}{ 3. Comorbidades } \\
\hline \multicolumn{2}{|l|}{ HAS } \\
\hline Sim & $35,19 \%(38)$ \\
\hline Não & $64,81 \%(70)$ \\
\hline \multicolumn{2}{|l|}{ IC } \\
\hline Sim & $7,41 \%(8)$ \\
\hline Não & $92,59 \%(100)$ \\
\hline \multicolumn{2}{|l|}{ 4.Exames solicitados } \\
\hline \multicolumn{2}{|l|}{ ECG } \\
\hline Sim & $8,33 \%(9)$ \\
\hline Não & $91,67 \%(99)$ \\
\hline \multicolumn{2}{|l|}{ Laboratoriais para Doença de Chagas } \\
\hline Sim & $0 \%(0)$ \\
\hline Não & $100 \%(108)$ \\
\hline \multicolumn{2}{|l|}{ 5. Diagnóstico de Doença de chagas } \\
\hline Sim & $0 \%$ \\
\hline Não & $100 \%(108)$ \\
\hline
\end{tabular}

Fonte: Prontuários Unidade de Saúde Rio do Sal. HAS: Hipertensão Arterial Sistêmica, IC: Insuficiência Cardíaca.

Do total de pacientes atendidos na Unidade de saúde do Rio do Sal, o eletrocardiograma foi solicitado em apenas $8,33 \%$ (9) dos casos e em $88,9 \%$ (8) deles o resultado do exame foi normal. Somente em 11,1\% (1) dos eletrocardiogramas verificou-se alterações (sobrecarga ventricular, e alteração de repolarização). Foi constatado ainda, que em nenhum dos prontuários havia solicitação de exames laboratoriais para a doença de Chagas e que em nenhum dos 108(100\%) prontuários 
constavam a informação de que os pacientes apresentavam sorologia positiva para doença de Chagas, ou seja, não havia nenhum diagnóstico de Doença de Chagas na comunidade (Tabela 4).

\section{Discussão}

A DC é endêmica em alguns locais do Brasil com destaque para a região Nordeste, pelo fato de possuir grande quantidade de espécies de vetores transmissores dessa parasitose (Matos, 2014). Segundo o SINAN (Sistema de Informação de Agravos de Notificação), no período de 2013 a 2017, foram notificados 396 casos suspeitos de doença de Chagas aguda (DCA) (Brasil, 2018). Dos casos notificados nesse período, as macrorregiões com maior número de notificações foram a Norte, Leste e Centro Leste. Segundo o boletim epidemiológico da Bahia e o MS (Ministério da Saúde), a cidade de Paulo Afonso possui risco médio de transmissão vetorial para Doença de Chagas (Brasil, 2018).

Neste trabalho foi relatado o perfil epidemiológico da DC na população rural do Rio do Sal, município de Paulo Afonso/BA e que se encontra na região norte da Bahia. A pesquisa contou com a participação de 64 pessoas e um problema encontrado no desenvolvimento da pesquisa foi que inúmeras casas se encontravam fechadas durante as visitas, e muitas outras estavam em construção sem habitação, sendo assim, o número de entrevistas foi realizado em um maior número possível de residências. Além desse problema houve grande resistência por parte da população em participar da pesquisa e que foi relacionado com a falta de confiança para com o serviço de saúde e o baixo conhecimento da população.

É sabido que a doença de chagas teve seus primeiros registros realizados na população rural e nos anos até 1950, a doença de Chagas era conhecida como endemia predominantemente rural (World Health Organization, 2015; Dias, 2013). Embora tenha havido uma modificação no padrão de distribuição do vetor e da doença também para áreas urbanas, as vigilâncias entomológicas indicam a presença desse inseto vetor com maior predominância ainda em áreas rurais (Dias, 2013; Brasil, 2014). Apesar de na pesquisa ter sido observado que $100 \%$ dos entrevistados residiam em casas de alvenaria, muitos relataram já ter encontrado o inseto triatomíneo em suas casas (Tabela 1). Dados da literatura relatam que os barbeiros são encontrados principalmente em casas de taipa, onde conseguem se esconder com maior facilidade (Araújo, Rodrigues, Rezende, Villela \& Borsuk, 2015). No entanto, informações atuais também ressaltam uma maior adaptação desses barbeiros em novas localizações, incluindo casas de alvenaria e em zonas urbanas (Dias et al., 2016; Dias, 2013).

No quesito escolaridade foi percebido que boa parte dos indivíduos não tem o ensino fundamental completo e que a maioria dos entrevistados possuía um baixo grau de escolaridade, o que reflete diretamente no nível de conhecimento dos mesmos quanto ao vetor transmissor e a DC. Gontijo, Rocha e Oliveira (1996) em seu estudo demonstrou que o nível de escolaridade está ligado ao maior número de indivíduos infectados e ao maior desconhecimento destes sobre a DC, o que também foi observado na presente pesquisa.

Observando-se as comorbidades encontradas na pesquisa, as doenças circulatórias, como hipertensão arterial sistêmica e doenças coronarianas, apresentaram prevalência de 42,6\% (46 pessoas); observando-se as comorbidades encontradas em estudo prévio por Carvalho et al (2003) as doenças circulatórias (hipertensão arterial sistêmica e doenças coronarianas) apresentavam prevalência de $33 \%$ nos pacientes com DC diagnosticadas, sendo essas as comorbidades mais encontradas nos chagásicos. Dos prontuários analisados na unidade de saúde, apenas 8,33\% (9) possuíam eletrocardiograma (ECG), e destes, somente um apresentava alteração, onde o laudo descrevia hipertrofia ventricular e alteração de repolarização.

O Consenso Brasileiro sobre a Doença de Chagas define a cardiopatia chagásica crônica pela presença de anormalidades eletrocardiográficas sugestivas de comprometimento cardíaco, em indivíduo com ou sem sintomas (Dias et al., 2016). Entretanto, os achados eletrocardiográficos no presente estudo não estão de acordo com os dados da literatura (Dias $e t$ al., 2016; Coura et al., 2013; Menezes, et al., 2011). Rosembaum e Alvarez (1955) encontraram em seu estudo com pacientes positivos para DC, prevalência de alterações eletrocardiográficas como o bloqueio de ramo direito $(55,7 \%)$ e extrassístoles 
ventriculares (36\%). Todavia, as alterações no ECG encontradas na população rural do Rio do Sal estão mais associadas com hipertensão arterial e insuficiência coronariana e, seriam elas, alterações da repolarização ventricular, hipertrofia ventricular e inatividade elétrica, o que não representam achados característicos na doença de Chagas (Dias et al., 2016; Coura et al., 2013; Rosembaum \& Alvarez, 1995).

A falta de investigação para a doença de Chagas foi evidenciada pela ausência de informações no prontuário médico (Tabela 4). Não foi observada em nenhum dos 108 prontuários a solicitação de sorologias para DC em qualquer paciente, embora alguns deles tenham apresentado sintomas cardiovasculares importantes, era de se esperar que tais sorologias fossem solicitadas, uma vez que a área possui informações relevantes sobre a presença do inseto triatomíneo.

A soroprevalência da população rural do país, feita por inquérito sorológico entre 1975 e 1980, era de 4,2\% para a população rural brasileira (Dias et al., 2016; Dias et al., 2000; Dias, 2000b). Porém, em recentes estudos, essa prevalência declinou para aproximadamente $0,2 \%$. E embora o controle atual da transmissão da doença de Chagas tenha resultado em queda da incidência de novos casos nos últimos anos, isto não significa que houve erradicação da Doença de Chagas. Ainda existem desafios a serem superados, principalmente em termos da assistência médica para os indivíduos subdiagnosticados, reforçando assim a importância da vigilância epidemiológica (Dias et al., 2016; Dias, 2000b).

\section{Conclusão}

Fica evidente a necessidade de uma investigação sorológica nessa população, uma vez que a região é endêmica para o vetor transmissor da doença de Chagas, descrita tanto por dados da Unidade de Controle de Zoonoses (UCZ) quando pelos próprios moradores que responderam em entrevista já ter encontrado o inseto em suas residências. Portanto, é se suma importância uma pesquisa diagnóstica por meio de exames confirmatórios, uma vez que podem existir portadores da DC nesta comunidade que ainda não foram detectados pelo Sistema Único de Saúde, e dessa maneira contribuir para que sejam notificadas e assim, desenvolver formas de promoção de saúde e controle da doença de Chagas.

Afim de alcançar novos resultados, é necessária a realização de novos trabalhos nesta população, e nas populações adjacentes à comunidade Rio do Sal, envolvendo a pesquisa sorológica para investigação de possíveis casos subnotificados. Além disso é também importante realizar estudos que investiguem os triatomíneos desta região, para identificação de possíveis agentes potencialmente infectados e infectantes.

\section{Referências}

Amorim, D. S. \& Costa, M. S. F. (2021). Tendência da mortalidade por doença de Chagas na Bahia: Entre os anos de 2008 a 2018 . Research, Society and Development. (10)5.

Araújo, A. D., Rodrigues, S. C., Rezende, A. F. S, Villela, M. M. \& Borsuk, S. (2015). Soroprevalência de infecção humana por Trypanosoma cruzi em uma área rural do sul do Brasil. Revista de Patologia Tropical. (44)4, 423-431.

Bozelli, C. E., Araújo, S. M., Guilherme, A. L. F. \& Gomes, L. F. (2006). Perfil clínico-epidemiológico de pacientes com a Doença de Chagas no Hospital Universitário de Maringá, Paraná, Brasil. Cadernos de Saúde Pública. (22)5, 1027-1034.

Brasil, Ministério da Saúde. (2014). Secretaria de Vigilância em Saúde. Guia de Vigilância em Saúde. Ministério da Saúde.

Brasil, Secretaria de Saúde do estado da Bahia. (2018, janeiro). Boletim epidemiológico da Doença de Chagas. Secretaria do estado da Bahia. (1). Available from: http://www.saude.ba.gov.br/wpcontent/uploads/2017/11/2018-Boletim-epidemiolÃgico-DoenÃßas-de-Chagas-n.-01.pdf

Carvalho, M. E., Silva, R. A., Barata, J. M., Domingos, M. F., Ciaravolo, R. M. \& Zacharias, F. (2003) Chagas' disease in the southern coastal region of Brazil. Rev Saúde Pública. (37)1, 49-58.

Coura, J., Viñas, P., Soares, L., Sousa, A. \& Xavier, S. (2013). Morbidity of Chagas heart disease in the microregion of Rio Negro, Amazonian Brazil: a casecontrol study. Memórias do Instituto Oswaldo Cruz. (108)8, 1009 - 1013. http://www.scielo.br/scieloOrg/php/articleXML.php?lang=ptpid=S007402762013000801009

Dias, J. (2000). Participação, descentralização e controle de endemias no Brasil. Doenças endêmicas: abordagens sociais, culturais e comportamentais. Editora Fiocruz. 269-97. 
Research, Society and Development, v. 10, n. 11, e477101119901, 2021

(CC BY 4.0) | ISSN 2525-3409 | DOI: http://dx.doi.org/10.33448/rsd-v10i11.19901

Dias, J., Ramos JR, A., Gontijo, E., Luquetti, A., Shikanai-Yasuda, M., Coura, J., Torres, R., Melo, J., Almeida, E., Oliveira Jr, W., Silveira, A., Rezende, J., Pinto, F., Ferreira, A., Rassi, A., Fragata Filho, A., Sousa, A., Correia Filho, D., Jansen, A., Andrade, G., Britto, C., Pinto, A., Rassi Jr, A., Campos, D., AbadFranch, F., Santos, S., Chiari, E., Hasslocher-Moreno, A., Moreira, E., Marques, D., Silva, E., Marin-Neto, J., Galvão, L., Xavier, S., Valente, S., Carvalho, N., Cardoso, A., Silva, R., Costa, V., Vivaldini, S., Oliveira, S., Valente, V., Lima, M. \& Alves, R. (2016). II Consenso Brasileiro em Doença de Chagas, 2015. Epidemiologia e Serviços de Saúde, 25(esp), 7-86. https://dx.doi.org/10.5123/s1679-49742016000500002

Dias, J. C. (2000). Epidemiological surveillance of Chagas disease. Cad Saúde Pública. (16)2, 43-59.

Dias, J. C. P. (2013) Human chagas disease and migration in the context of globalization: some particular aspects. J Trop Med.

Dias, J. C. P., Machado, E. M. M., Fernandes, A. L. \& Vinhaes, M. C. (2000). Esboço geral e perspectivas da doença de Chagas no Nordeste do Brasil. Cad Saúde Pública. (16)2, $13-34$.

Fontelles, M. J., Simões, M. G., Farias, H. S. \& Fontelles, R. G. S. (2009). Metodologia da pesquisa científica: diretrizes para a elaboração de um protocolo de pesquisa. Revista Paraense de Medicina. (23)3, 69-76.

Gontijo, E. D., Rocha, M. O. C. \& Oliveira, U. T. (1996). Perfil clínico-epidemiológico de chagásicos atendidos em ambulatório de referência e proposição de modelo de atenção ao chagásico na perspectiva do SUS. Revista da Sociedade Brasileira de Medicina Tropical. (29)2, 101 - 108. http://www.scielo.br/scieloOrg/php/articleXML.php?lang=enpid=S0037-86821996000200002.

Lima, R. S., Teixeira, A. B. \& Lima, V. L. S. (2019). Doença de Chagas: uma atualização bibliográfica. Revista Brasileira de Análises Cínicas. (51)2, 103106.

Matos, C. S. (2014). Doença de Chagas em Bambuí: Estado atual e vigilância [Programa de Pós-graduação em Ciências da Saúde]. Fundação Oswaldo Cruz, Belo Horizonte, MG, Brasil. http://www.cpqrr.fiocruz.br/texto-completo/T77.pdf.

Menezes, C., Costa, G. C., Gollob, K. J. \& Dutra, W. O. (2011) Clinical aspects of Chagas disease and implications for novel therapies. Drug Dev Res. (72)6, $471-479$.

Organization WH (2015). Chagas disease in Latin America: an epidemiological update based on 2010 estimates. (2015). Releve epidemiologique hebdomadaire, 90(6), 33-43.

Porfírio, D. M., Lobato, E. S. D., Trindade, G. P. \& Araújo Filho, G. G. (2020). Prevalência de Doença de Chagas em Idosos no Estado do Pará: Uma Análise Retrospectiva. Brazilian Journal of Health Review. 3(4), 9142-9152.

Rosembaum, M. B. \& Alvarez, A. J. (1955). The eletrocardiogram in chronic chagasic myocarditis. Am Heart J. (50)4, 492-527.

Teixeira, R. B. \& Oliveira, S. M. C. (2015). Perfil de pacientes portadores de Doença de Chagas em Rio Branco, Acre, Brasil. Rev Soc Bras Clin Med. (13)4, $262-265$. 